Supplementary Information

\title{
Machine-Learning Enabled Exploration of Morphology Influence on Wire-Array Electrodes for Electrochemical Nitrogen Fixation
}

\author{
Benjamin B. Hoar ${ }^{1}$, Shengtao Lu${ }^{1}$, Chong $\mathrm{Liu}^{1,2, *}$ \\ ${ }^{1}$ Department of Chemistry and Biochemistry, ${ }^{2}$ California NanoSystems Institute, University of \\ California, Los Angeles, Los Angeles, CA 90095, USA \\ * To whom correspondence may be addressed. Email: chongliu@chem.ucla.edu
}




\section{Table of Contents}

\section{Materials and Methods}

$\begin{array}{ll}\text { Finite element methods } & \text { S3 }\end{array}$

$\begin{array}{ll}\text { Model selection and training } & \text { S4 }\end{array}$

$\begin{array}{ll}\text { Evaluation of model performance } & \text { S5 }\end{array}$

The procedure of comparing simulation duration $\quad$ S5

$\begin{array}{ll}\text { Local and global electrode optimization } & \text { S6 }\end{array}$

\section{Supplementary Notes}

$\begin{array}{ll}\text { Derivation of finite element methods } & \text { S7 }\end{array}$

$\begin{array}{ll}\text { Comment on hardware limitations } & \text { S9 }\end{array}$

\section{Supplementary Figures}

$\begin{array}{ll}\text { Figure S1 Representative Tafel plots for the FEM simulations } & \text { S10 }\end{array}$

Figure S2 Outputs of literature catalysts optimized for F.E. or $i_{\text {total }} \quad$ S11

$\begin{array}{ll}\text { Figure S3 Feature importance analysis for the prediction of } i_{\text {total }} & \mathrm{S} 12\end{array}$

Figure S4 Comparison of trends for predictions of electrodes in morphology sensitive region S13

Figure S5 Feature-by-feature trends in morphology sensitive regions $\quad$ S14

Figure S6 Reactant concentration gradients for catalyst at $\eta=0.3 \mathrm{~V}$ vs. RHE S15

Figure S7 Reactant concentration gradients for catalyst at $\eta=0.5 \mathrm{~V}$ vs. RHE S16

Figure S8 Reactant concentration gradients for catalyst at $\eta=0.7$ V vs. RHE $\quad$ S17

Figure S9 Proton concentration gradient for catalyst discussed in Figure $3 \quad$ S18

\section{Supplementary Tables}

$\begin{array}{ll}\text { Table S1 Invariant parameters of FEM simulation } & \text { S19 }\end{array}$

$\begin{array}{ll}\text { Table S2 Ranges of parameters explored in FEM simulations } & \text { S20 }\end{array}$

$\begin{array}{ll}\text { Table S3 Hyperparameters of MLPNN models } & \text { S21 }\end{array}$

$\begin{array}{ll}\text { Table S4 Specifications of hardware } & \text { S22 }\end{array}$

$\begin{array}{ll}\text { Table S5 Details of MLPNN optimized electrodes for literature catalysts } & \text { S23 }\end{array}$

Table S6 Details of electrode morphologies circled in Figure 2A $\quad$ S24

$\begin{array}{ll}\text { Supplementary References } & \text { S25 }\end{array}$ 


\section{Materials and Methods}

\section{Finite Element Methods}

Finite element methods (FEM) for simulating nitrogen reduction reaction (NRR) and hydrogen evolution reaction (HER) activity of electrodes were conducted using COMSOL Multiphysics (Version 5.3) (Figure 1A to 1C). The basis of the FEM model used here was reported previously as a study of micro-wire NRR electrode efficacy and its concomitant mass transport complications. ${ }^{1}$ Electrode activity was calculated assuming aqueous non-oxidizing strong acid electrolyte with a concentration of $0.1 \mathrm{M}$ at $293 \mathrm{~K}$ with other invariant FEM simulation parameters shown in Table S1. ${ }^{2-4}$ Other parameters of the FEM were variable and consisted of morphology descriptors (wire geometry: periodicity $p$, length $l$, diameter $d$ ), applied potential ( $\eta$ vs. Reversible Hydrogen Electrode, RHE), and catalytic activity descriptors (exchange current densities of

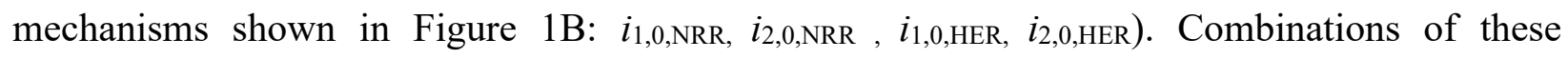
descriptors were implemented using parametric sweep (Table S2).

The 3D model constructed for the above descriptors also accounted for the diffusion layer relevant to the electrochemical NRR system, the layer being defined by the distance between the highest point of the micro-wire and the diffusion layer boundary. This FEM model consisted of a micro-wire whose unit cell's base was defined by the periodicity of the electrode wires and whose height was defined by the length of the microwire plus the diffusion layer thickness. Of this model, one surface represented the electrode and the one opposite to this surface represented the diffusion layer boundary, beyond where reagents existed in their bulk electrolyte concentrations. For all other surfaces, periodic boundary conditions were applied. Migration effects were assumed to be minimal due to supporting electrolyte effects and only diffusion was considered.

The nitrogen fixation reaction in acidic solution is given by:

$$
\mathrm{N}_{2}(\mathrm{aq})+6 \mathrm{e}^{-}+8 \mathrm{H}^{+} \rightarrow 2 \mathrm{NH}_{4}^{+}(\mathrm{aq})
$$

The kinetic rate description of the reaction is given by equation S8 provided in Supplementary Note 1 . The parameters explored unique to this reaction were $i_{1,0, \mathrm{NRR}}$ and $i_{2,0, \mathrm{NRR}}$. Applied potential and morphological parameters were also explored in combination with these kinetic descriptors and could also influence the kinetics of the reaction.

The hydrogen evolution reaction in acidic solution is given by:

$$
2 \mathrm{H}^{+}(\mathrm{aq})+2 \mathrm{e}^{-} \rightarrow \mathrm{H}_{2}(\mathrm{aq})
$$


The kinetic rate description of the reaction is given by equation S12 provided in Supplementary Note 1 . The parameters explored unique to this reaction were $i_{1,0, \mathrm{HER}}$ and $i_{2,0, \mathrm{HER}}$. Other swept parameters could influence HER kinetics as described for NRR.

The ranges of exchange current densities for NRR and HER activity used were $10^{-8}$ to $10^{-3}$ $\mathrm{mA} / \mathrm{cm}^{2}$ based on previously reported experimental data. ${ }^{5-6}$

The total current density $\left(i_{\text {total }}\right)$ is calculated as:

$$
i_{\text {total }}=\frac{I_{\text {total }}}{A}=\frac{I_{\mathrm{NRR}}+I_{\mathrm{HER}}}{A}=\frac{\int_{A} i_{\text {loc, NRR }} \mathrm{d} A+\int_{A} i_{\text {loc, } \mathrm{HER}} \mathrm{d} A}{A}
$$

here, $A$ denotes the electrode surface area and $I_{\mathrm{otal}}, I_{\mathrm{NRR}}$, and $I_{\mathrm{HER}}$ denote total current passing through the electrode, the current used for NRR and the current used for HER, respectively.

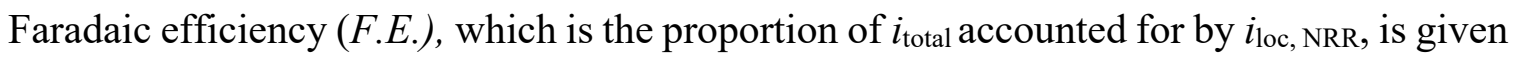
by:

$$
F . E .=\frac{I_{\mathrm{NRR}}}{I_{\text {total }}}=\frac{\int_{A} i_{\text {loc, NRR }} \mathrm{d} A}{\int_{A} i_{\text {loc, NRR }} \mathrm{d} A+\int_{A} i_{\text {loc, } \mathrm{HER}} \mathrm{d} A}
$$

\section{Model selection and training}

The implementation of all machine-learning code was done on Google Cloud Platform's (GCP) AI Platform, with code specifically deployed with the beta version of their JupyterLab Notebook implementation. This allowed for virtual machines with adjustable power depending on the demands of the contemporary goal. All machine-learning methods were implemented using the scikit-learn machine-learning package (version 0.21 .2 ) with data management largely provided by the pandas software package (version 0.24.2) as well as graphing provided by the matplotlib software package (version 3.1.0). For convenience all of our machine-learning studies arbitrarily operate with a geometric resolution of $1 \mu \mathrm{m}$, which can be improved in future investigations.

Data was imported from FEM results in the form of CSV files and combined into library of data. Before model development began, a random 30\% of the data was split from the overall dataset and set aside for later model validation. The rest of the data was normalized (scikit-learn StandardScaler) and scaled (scikit-learn MinMaxScaler) in preparation for implementation of machine learning algorithms. Model selection proceeded via a script combining a list of candidate models with coarse grid search methods to provide options for final model deployment. Model success was evaluated using a mean squared error (MSE) loss function and built-in three-fold cross-validation. 
Upon completion of model selection procedures, scikit-learn's multi-layer perceptron (MLPNN) neural network provided the most promising results. The hyperparameters of the MLPNN is displayed in Table S3. A finer grid search of MLPNN hyper-parameters was conducted to establish the final model with the early-stopping hyper-parameter set to "true" as our primary means to avoid model over-fitting.

\section{Evaluation of model performance}

The quality of the models' fit was further validated using the $30 \%$ of data separated premodel selection. Model validity was measured using the MSE of our neural network predicted values versus FEM predictions. Testing data and training data covered the same parameter space and provided model evaluation as it related to parameters previously seen by the model (Figures $1 \mathrm{E}-\mathrm{F})$.

A sensitivity analysis was performed to determine the individual importance of each feature by one-at-a-time randomization of features, training the model with dataset containing the randomized feature, and re-evaluation of model predictive power (Figure 3A). Importance was determined by comparing the MSE of the data-altered model and the model trained on the unaltered data.

Evaluation of model's ability to predict electrode activity in regions with high morphology influence was explored by selecting a catalyst (defined by values: $i_{1,0, \mathrm{NRR}}=10^{-6} \mathrm{~mA} / \mathrm{cm}^{2}, i_{2,0, \mathrm{NRR}}$ $\left.=10^{-3} \mathrm{~mA} / \mathrm{cm}^{2}, i_{1,0, \mathrm{HER}}=10^{-4} \mathrm{~mA} / \mathrm{cm}^{2}, i_{2,0, \mathrm{HER}}=10^{-6} \mathrm{~mA} / \mathrm{cm}^{2}\right)$ and potential value $(\eta=-0.5 \mathrm{~V}$ vs. RHE) where a wide range of Faradaic efficiency values were present as a function of morphology change alone (Figure 3C, S5). Data was generated by FEM and MLPNN and compared for correlation in trend (Figure S4). Ranges of morphological parameters explored were determined by literature and simulation results and the accuracy of predictions was measured on a global (Figure S4) and parameter-by-parameter basis (Figures S4-5).

\section{The procedure of comparing simulation duration}

The comparison of simulation durations between the FEM and MLPNN approaches (Figure 1G) were conducted on an Intel® Core ${ }^{\mathrm{TM}}$ i7-7800 CPU @ 3.50 GHz with 32 GB of random access memory (RAM) and 6 central processing unit (CPU) cores with an NVIDIA GeForce GTX 1060 3GB graphics processing unit (GPU) and a $1.4 \mathrm{GHz}$ Dual-Core i5 MacBook Air with 8 GB 
of RAM (MD760LL/B) for the FEM and MLPNN simulations, respectively (Table S4). Up to the order of $10^{3}$ predictions could be taken using the FEM procedure before exhausting the RAM, and up to the order of $10^{5}$ predictions could be made using the MLPNN before the same issue occurred.

\section{Local and global electrode optimization}

Global optimization (Figure 2A) was achieved by generating over 4,000,000 possible catalyst and morphology combinations via the neural-network approach and presenting the combinations predicted to have greater than $2 \mathrm{~mA} / \mathrm{cm}^{2}$ of $i_{\text {total }}$ and $90 \%$ of F.E. Literature-based catalyst information was derived using equations S8 and S12 (Supplementary note 1) in combination with the WebPlotDigitizer tool provided by Ankit Rohatgi at https://apps.automeris.io/wpd/ to extract data from published literature plots. The automeris.io application allowed the conversion of published graphical data into raw data. This data was then used to calculate NRR, HER, and F.E. data from published information. This data was sufficient to estimate the kinetic activity descriptors $\left(i_{1,0, \mathrm{NRR}}, i_{2,0, \mathrm{NRR}}, i_{1,0, \mathrm{HER}}\right.$, and $\left.i_{2,0, \mathrm{HER}}\right)$ necessary for application in the neural network models. Morphologies to maximize F.E., $i_{\text {total, }}$ or both simultaneously were predicted for each literature-derived catalyst (Figure $2 \mathrm{~B}, \mathrm{~S} 2) .^{7-11}$

\section{Generation of reactant concentration profiles}

Reactant profiles shown in Figure 3D and Figures S6-9 were generated using COMSOL Multiphysics 5.3. Upon solving for the current densities of the electrode materials, two-dimensional concentration gradients bisecting the wire of the cell were plotted for both proton and nitrogen (as shown right). For a given grouping of wire concentration profiles, an absolute scale was presented for the reactant of interest, this can give the appearance of no reactant concentration profile where diffusion in not highly obstructed (i.e. Figure S6 far left panels); for these cases, the change in concentration of species from the top of the cell to the bottom was minimal, so a mostly monochromatic concentration profile results from the simulation.

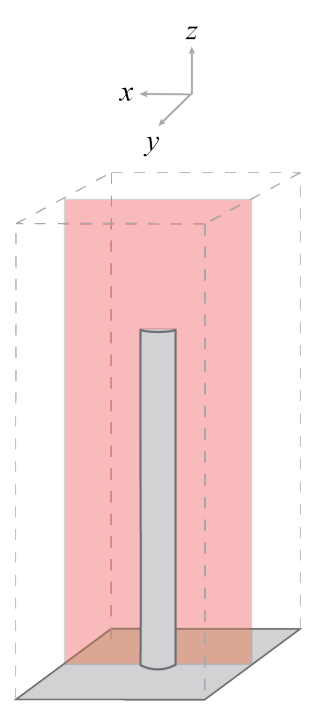




\section{Supplementary Notes}

\section{Note 1: Summary of FEM equations}

In our FEM model for electrocatalytic NRR, the kinetics of electrochemical nitrogen reduction consider the following steps (Figure 2B):

$$
\begin{array}{lll}
\text { Electron transfer (ET) } & \mathrm{N}_{2}(a q)+e^{-} \leftrightharpoons \mathrm{N}_{2}^{\bullet-}(a d) & k_{f 1}(\eta), k_{b 1}(\eta) \\
\text { Proton transfer (PT) } & \mathrm{N}_{2}^{\bullet-}(a d)+\mathrm{H}^{+}(a q) \rightarrow \mathrm{HN}_{2}(a d) & k_{f 2}
\end{array}
$$

Here, $k_{f l}$ and $k_{b l}$ are forward and backward rate constants - functions of overpotential - for the ET step, respectively. $k_{f 2}$ is the rate constant of PT step. States are given by $a q$, aqueous; $a d$, adsorbed to catalyst surface. At steady state, the concentration of $\mathrm{N}_{2}^{--}(a d)$ remains constant. Using this information, the local current density of nitrogen fixation $i_{l o c, \mathrm{NRR}}$ can be written as:

$$
i_{\mathrm{loc}, \mathrm{NRR}}=-n F \frac{k_{f 1,0}\left[\mathrm{~N}_{2}(a q)\right] \exp \left(\frac{\alpha_{C} F \eta}{R T}\right)}{\frac{k_{b 1,0} \exp \left(\frac{\alpha_{C} F \eta}{R T}\right)}{k_{f 2,0}\left[\mathrm{H}^{+}(a q)\right]}+1}
$$

Where $k_{f 1,0}$ and $k_{b 1,0}$ are rate constants at 0 over potential. $\alpha_{c}$ is the cathodic charge exchange coefficient, F is Faraday's constant, $\mathrm{T}$ is temperature in kelvin, and $\mathrm{R}$ is the gas constant. Further modification can be made to the equation resulting in our generalized NRR equation:

$$
i_{\mathrm{loc}, \mathrm{NRR}}=-\frac{i_{1,0, \mathrm{NRR}} \frac{\left[\mathrm{N}_{2}(a q)\right]}{{ }_{\mathrm{N}_{2}}(a q)} \exp \left(\frac{-\alpha_{C} F \eta}{R T}\right)}{1+\frac{i_{1,0, \mathrm{NRR}} C_{\mathrm{H}^{+}}{ }_{(\mathrm{aq})} \exp \left(\frac{\alpha_{C} F \eta}{R T}\right)}{i_{2,0, \mathrm{NRR}}\left[\mathrm{H}^{+}(a q)\right]}}
$$

Where $i_{1,0, \mathrm{NRR}}$ and $i_{2,0, \mathrm{NRR}}$ are the exchange current densities when the rate determining step (RDS) is ET and PT respectively (Figure S1A). $c_{\mathrm{N}_{2}(a q)}$ and $c_{\mathrm{H}^{+}(a q)}$ are the bulk concentrations of $\mathrm{N}_{2}$ and $\mathrm{H}^{+}$and $\left[\mathrm{N}_{2}(\mathrm{aq})\right]$ and $\left[\mathrm{H}^{+}(\mathrm{aq})\right]$ are the concentrations of species at the electrode surface.

The FEM equation can be discerned in a similar way, where we consider a VolmerHeyrovský mechanism: $:^{5,6}$

$$
\begin{array}{ll}
\text { Volmer: } & \mathrm{H}^{+}(a q)+e^{-} \leftrightharpoons \mathrm{H}(a d) \quad k_{f 3}(\eta), k_{b 3}(\eta) \\
\text { Heyrovksý: } & \mathrm{H}(a d)+\mathrm{H}^{+}(a q)+e^{-} \rightarrow \mathrm{H}_{2}(a q)
\end{array}
$$

Here, $k_{f 3}$ and $k_{b 3}$ are forward and backward rate constants - functions of overpotential - for the ET step, respectively. $k_{f 4}$ is the rate constant of the Heyrovský step. H (ad) represents surface-bound hydrogen atom. 
Applying steady-state conditions and potential dependency for the rate constants $k_{f 3}, k_{b 3}$, and $k_{f 4}$ we derive:

$$
i_{\mathrm{loc}, \mathrm{HER}}=-n F \frac{k_{f 3,0}\left[\mathrm{H}^{+}(a q)\right] \exp \left(\frac{-\alpha_{c} F \eta}{R T}\right)}{1+\frac{k_{b 3,0} \exp \left(\frac{2 \alpha_{c} F \eta}{R T}\right)}{k_{f 4,0}\left[\mathrm{H}^{+}(a q)\right]}}
$$

Here, $i_{l o c, \text { HER }}$ is the local hydrogen evolution current density. Similarly, this equation can be rewritten using the exchange current density notation relevant to our simulation:

$$
i_{\mathrm{loc}, \mathrm{HER}}=-\frac{\left.i_{1,0, \mathrm{HER} \frac{\left[\mathrm{H}^{+}(a q)\right]}{c} \exp \left(\frac{-\alpha_{c} F \eta}{R T}\right)}^{\mathrm{H}^{+}(a q)}\right)}{1+\frac{i_{1,0, \mathrm{HER}{ }_{\mathrm{H}^{+}}{ }_{(\mathrm{aq})}} \exp \left(\frac{2 \alpha_{c} F \eta}{R T}\right)}{i_{2,0, \mathrm{HER}}\left[\mathrm{H}^{+}(a q)\right]}}
$$

In this equation, $i_{1,0, \mathrm{HER}}$ and $i_{2,0, \mathrm{HER}}$ represent exchange current densities when the RDS is the Volmer and Heyrovský steps respectively (Figure S1B). 


\section{Note 2: Hardware Limitations}

In Figure 1G, the plotted curves showing the time to prediction comparison between FEM and MLPNN methods transition from solid to dashed lines. The transition indicates the number of electrodes predicted before human intervention is needed to further predict new electrodes for their respective methods. Because of RAM depletion (computer memory becomes exhausted), the data must be exported and predictions manually restarted, meaning $10^{3}$ and $10^{5}$ electrodes are easily predictable for the FEM and MLPNN, respectively. 
A

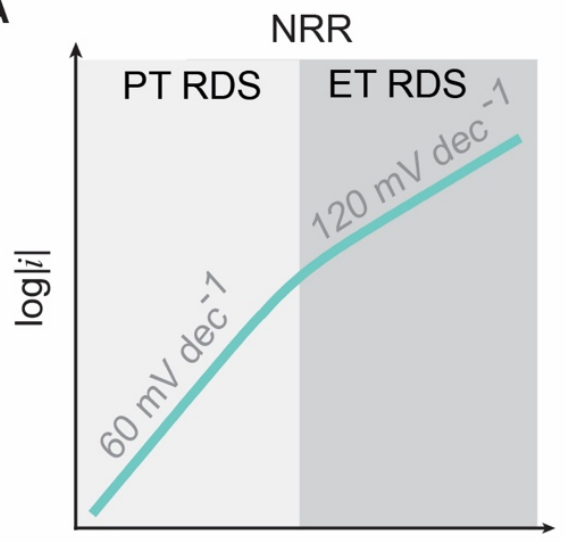

$|\eta|$
B

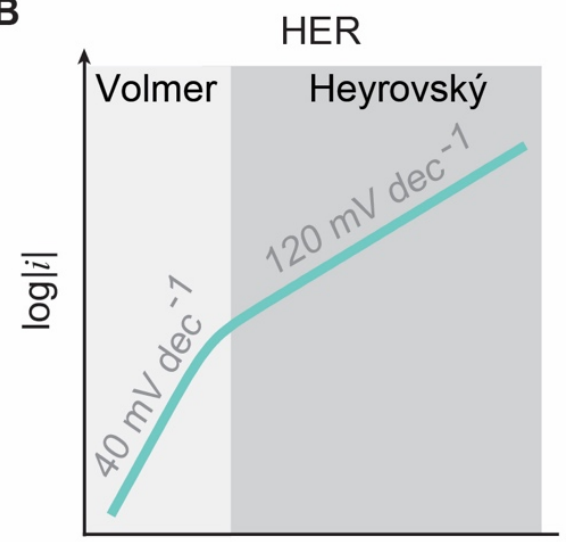

$|\eta|$

Figure S1. Representative $\log \left(i_{\text {total }}\right)$ versus $\eta$ (overpotential) plots of our FEM simulations that show the mechanistic dependence on overpotential of the two competing processes. For the NRR, the proton transfer step is rate limiting at low overpotentials as the slope of $60 \mathrm{mV} /$ decade implies an EC mechanism. At higher overpotentials, the electron transfer step becomes rate limiting as the slope of $120 \mathrm{mV} /$ decade implies an E mechanism. For the HER, a similar trend is shown except at low overpotentials the $40 \mathrm{mV} /$ decade slope implies an EE mechanism transitioning into an $\mathrm{E}$ mechanism at higher overpotentials. 
A

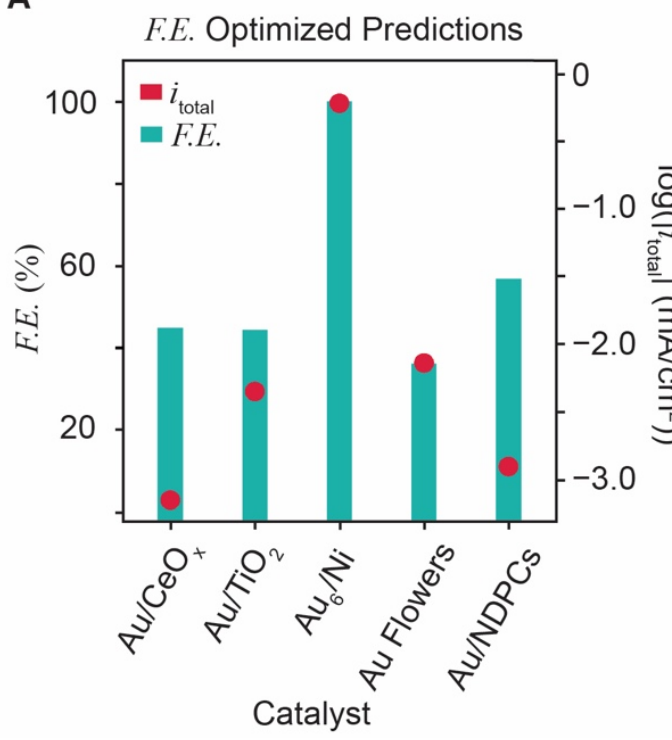

B

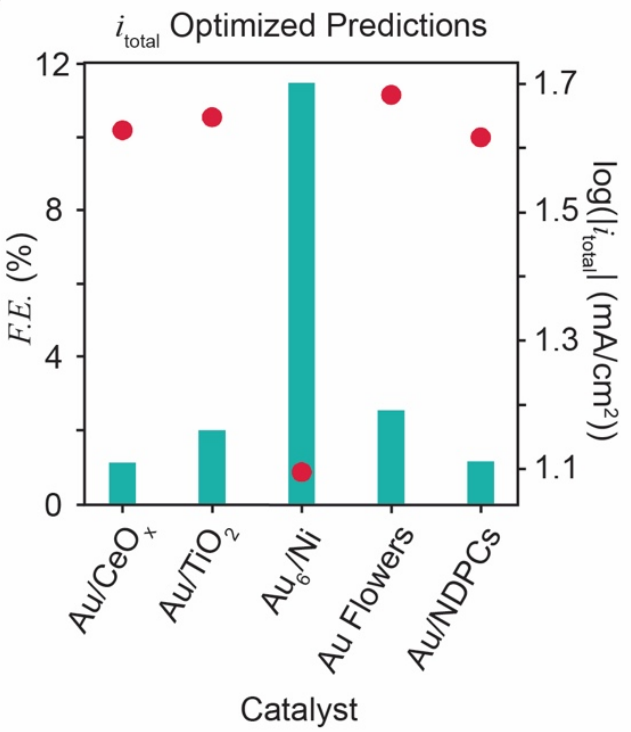

Figure S2. $i_{\text {total }}$ and F.E. versus catalyst outputs for literature-based materials. Electrodes are maximized for either F.E. (A) or $i_{\text {total }}(\mathbf{B})$. 


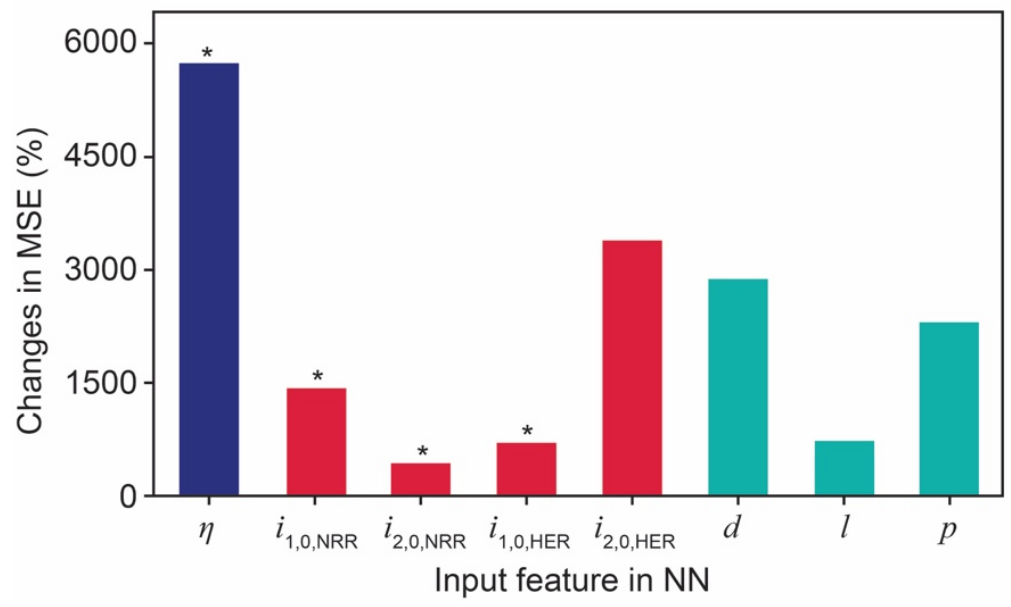

Figure S3. Change in MSE as a percentage versus input feature indicates relative feature importance values for the MLPNN tasked with predicting $i_{\text {total }}$. Bars marked with a "*" have been divided by a factor of 50 . The procedure is the same as that presented for the feature importance analysis for F.E. in Figure 3A. 

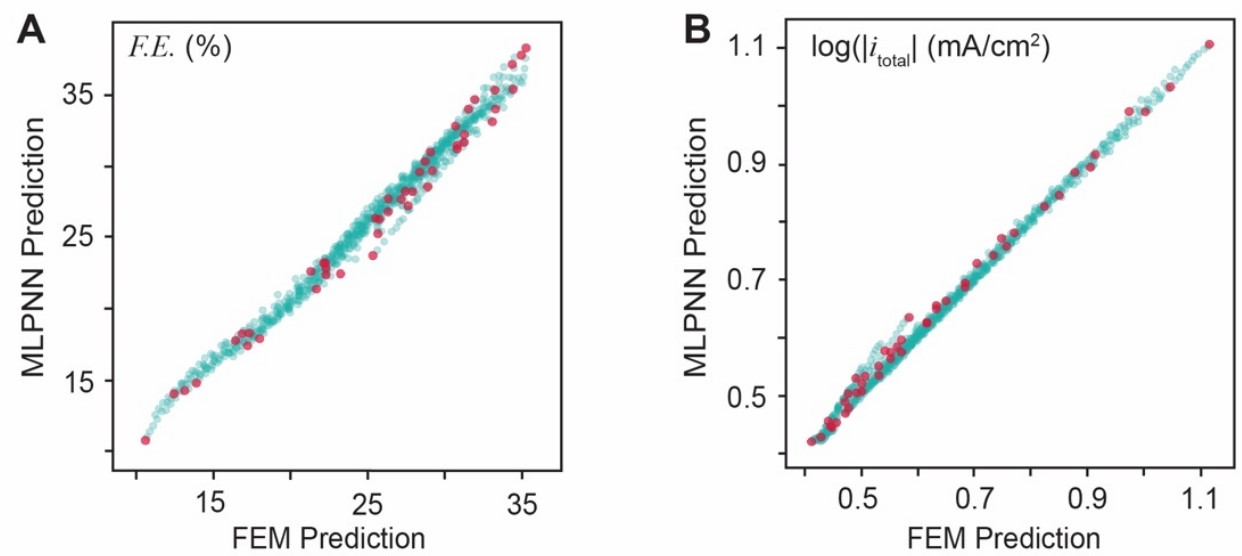

Figure S4. Correlation of FEM and MLPNN predictions for the expanded morphology set for the catalyst described by $i_{1,0, \mathrm{NRR}}=10^{-6}, i_{2,0, \mathrm{NRR}}=10^{-3}, i_{1,0, \mathrm{HER}}=10^{-4}$, and $i_{2,0, \mathrm{HER}}=10^{-6} \mathrm{~mA} / \mathrm{cm}^{2}(\mathrm{same}$ catalyst discussed in Figure 3). Red dots show morphologies seen by the original training data and green dots indicate never-before-seen morphologies. 

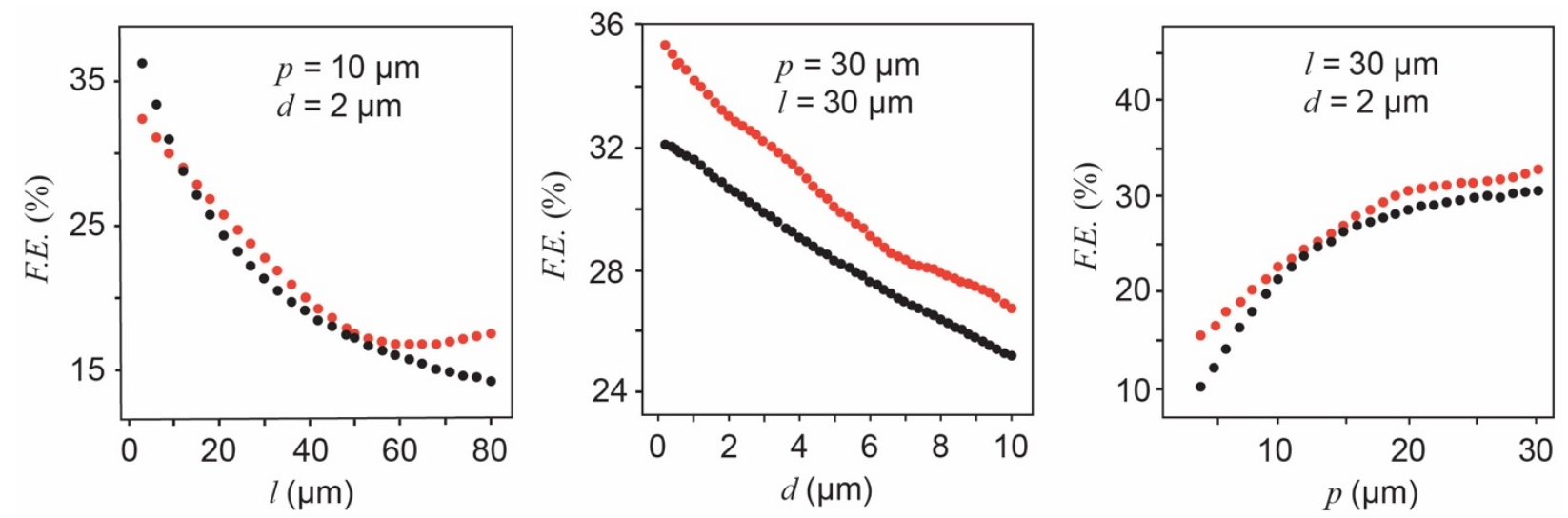

Figure S5. F.E. versus morphology-feature plots predicted by the MLPNN (red) and FEM (black) in a highly morphology-sensitive region. 


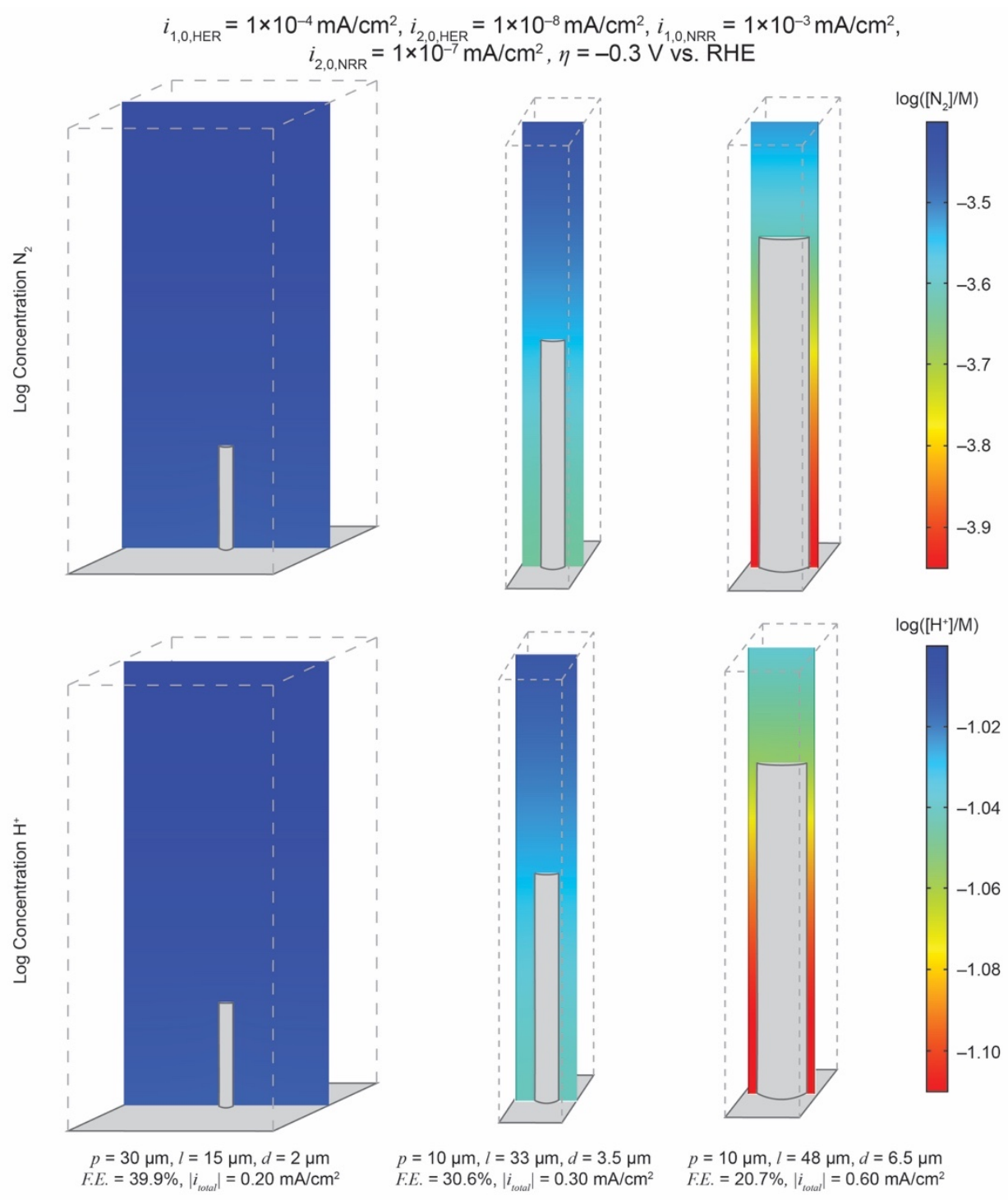

Figure S6. Reactant concentration profiles for a material with high morphology dependent Faradaic efficiency at an overpotential of $-0.3 \mathrm{~V}$. Nitrogen concentration profile above, proton concentration below. 


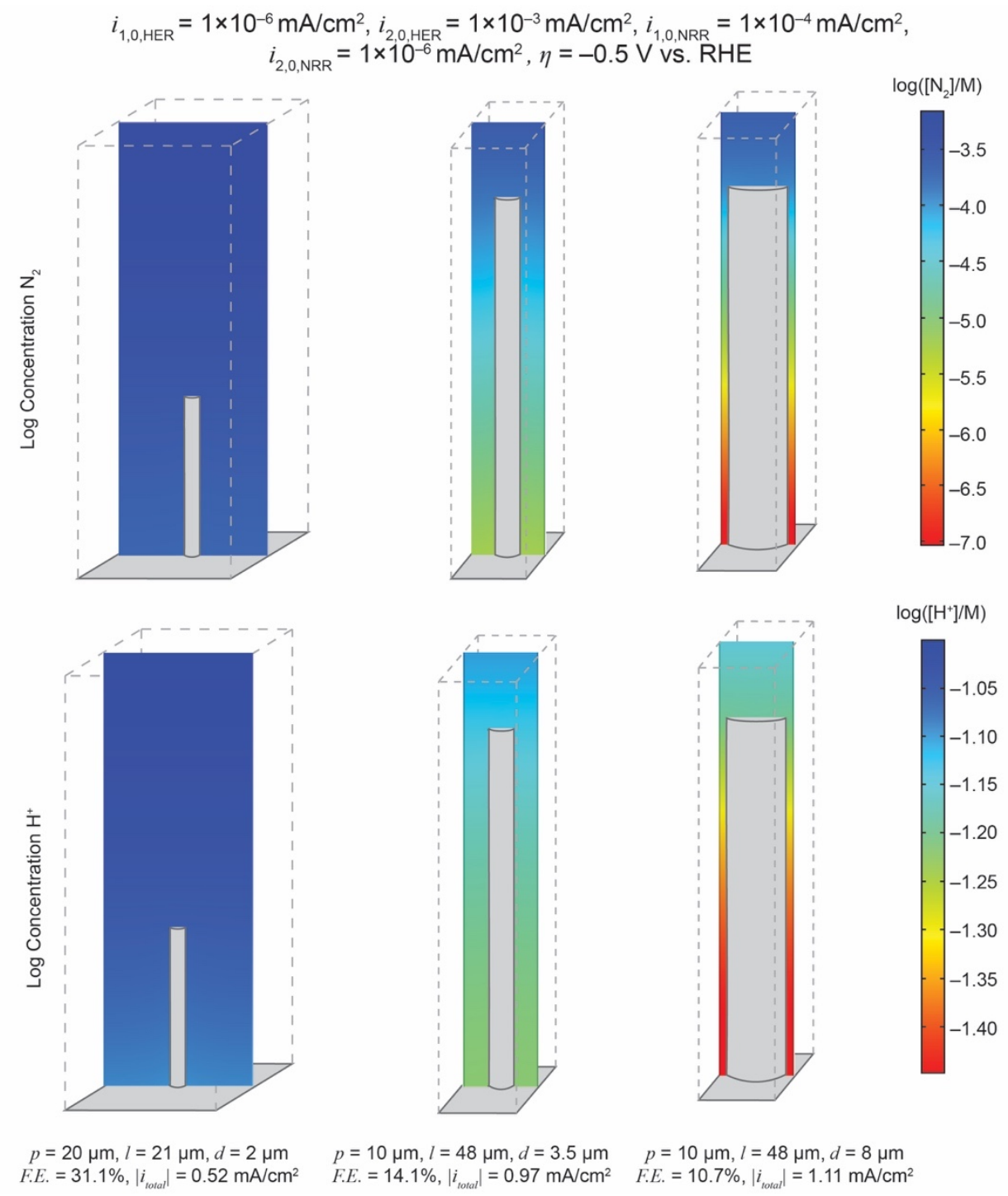

Figure S7. Reactant concentration profiles for a material with high morphology dependent Faradaic efficiency at an overpotential of $-0.5 \mathrm{~V}$. Nitrogen concentration profile above, proton concentration below. 


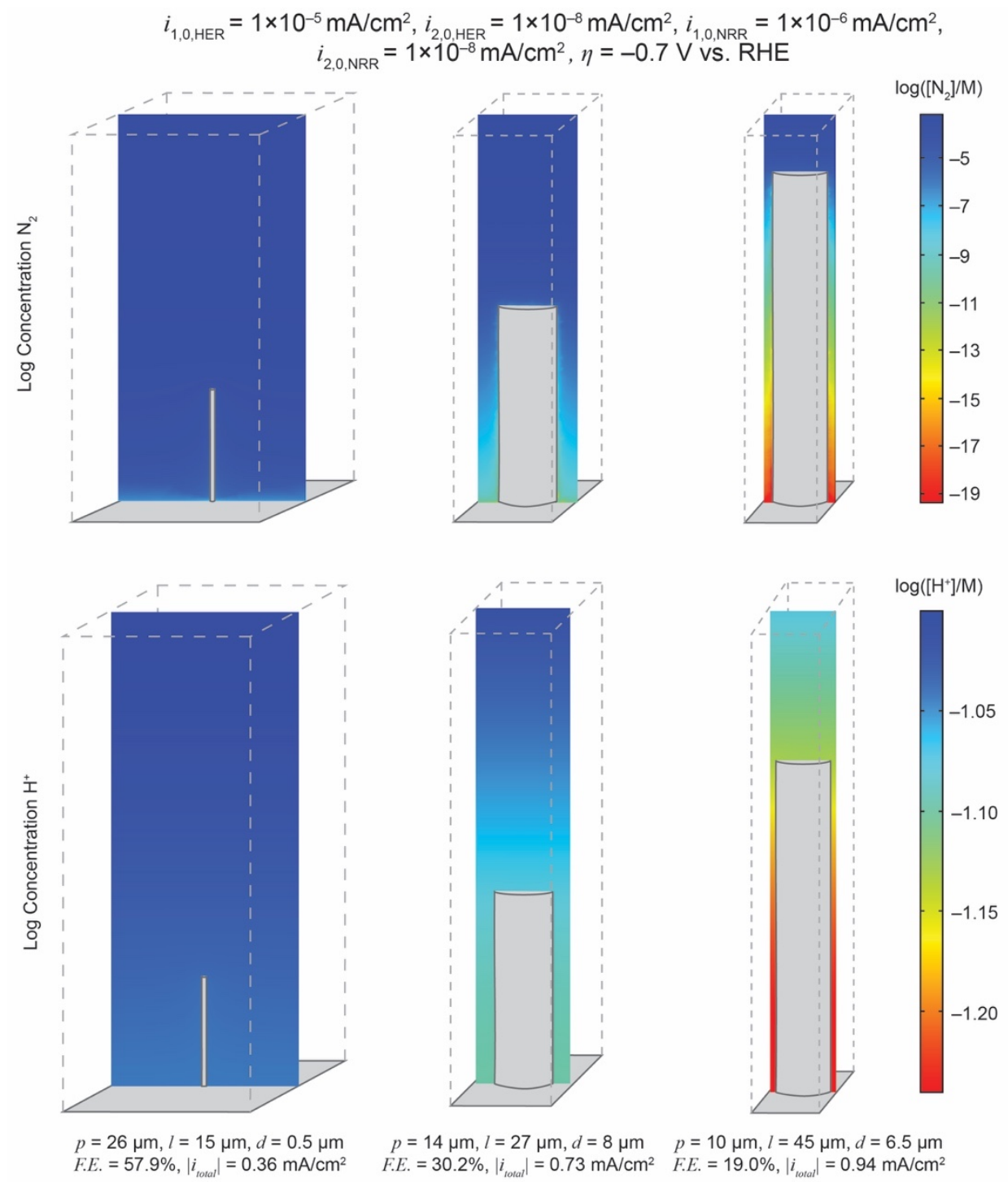

Figure S8. Reactant concentration profiles for a material with high morphology dependent Faradaic efficiency at an overpotential of $-0.7 \mathrm{~V}$. Nitrogen concentration profile above, proton concentration below. 


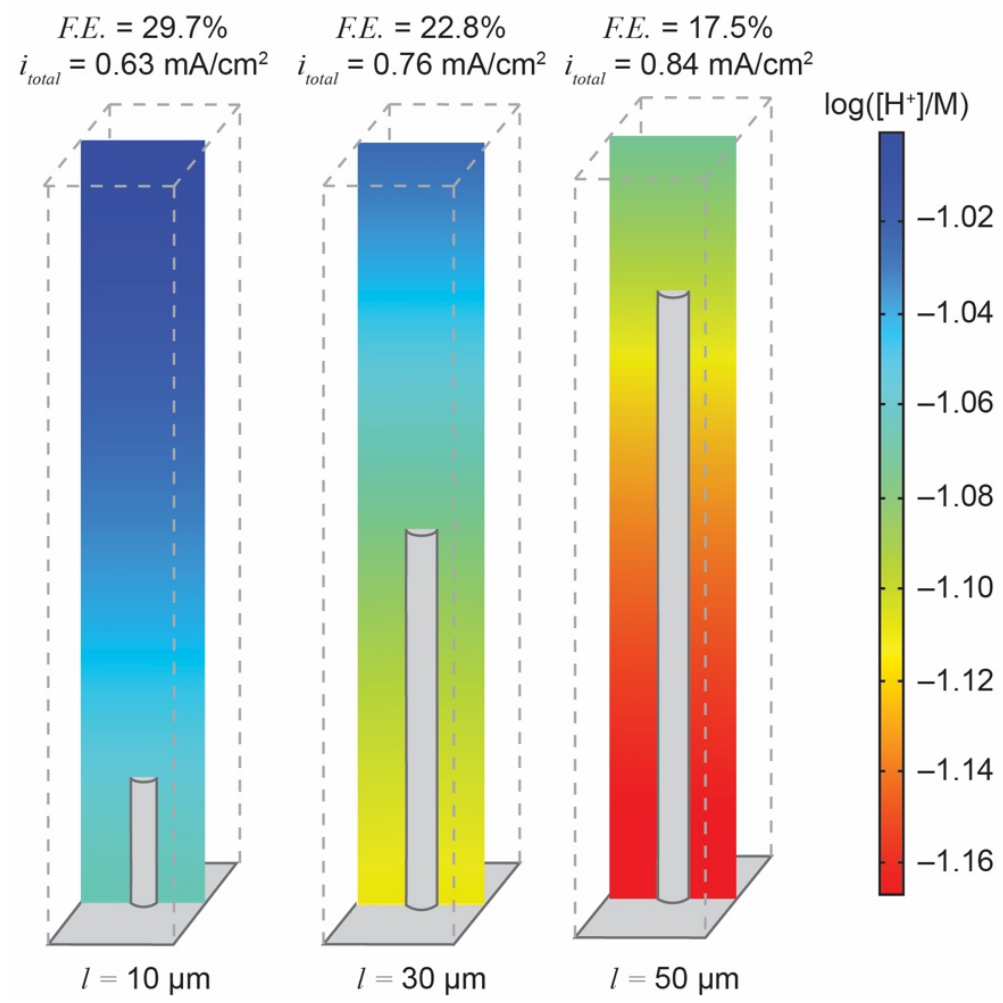

Figure S9. Proton concentration profiles for a catalytic material showing high Faradaic efficiency dependence on wire-array length. Nitrogen concentration gradient shown in Figure 3D. Kinetic descriptors of this system: $i_{1,0, \mathrm{NRR}}=10^{-6}, i_{2,0, \mathrm{NRR}}=10^{-3}, i_{1,0, \mathrm{HER}}=10^{-4}$, and $i_{2,0, \mathrm{HER}}=10^{-6} \mathrm{~mA} / \mathrm{cm}^{2}$ and $\eta=-0.5 \mathrm{~V}$ vs. RHE. 
Table S1. Invariant parameters of FEM simulation

\begin{tabular}{|c|c|}
\hline Parameter & Value \\
\hline Diffusion layer thickness & $53.5 \mu \mathrm{m}$ \\
\hline Diffusion coefficient of $\mathrm{N}_{2}, D_{\mathrm{N}_{2}}$ & $2.0 \times 10^{-9} \mathrm{~m}^{2} / \mathrm{s}$ \\
\hline Diffusion coefficient of $\mathrm{H}^{+}, D_{\mathrm{H}^{+}}$ & $1.9 \times 10^{-9} \mathrm{~m}^{2} / \mathrm{s}$ \\
\hline Bulk concentration of $\mathrm{N}_{2}, C_{\mathrm{N}_{2}}$ & $7.14 \times 10^{-4} \mathrm{~mol} / \mathrm{L}$ \\
\hline Bulk concentration of $\mathrm{H}^{+}, c_{\mathrm{H}^{+}}$ & $0.1 \mathrm{~mol} / \mathrm{L}$ \\
\hline Standard reduction potential of $\mathrm{N}_{2} / \mathrm{NH}_{4}^{+}, E_{\mathrm{N}_{2} / \mathrm{NH}_{4}^{+}}^{0}$ & $0.27 \mathrm{~V} \mathrm{vs.} \mathrm{RHE}$ \\
\hline Standard reduction potential of $\mathrm{H}^{+} / \mathrm{H}_{2}, E_{\mathrm{H}^{+}}^{0} / \mathrm{H}_{2}$ & $0 \mathrm{~V}$ vs. RHE \\
\hline Cathodic charge transfer coefficient, $\alpha_{\mathrm{c}}$ & 0.5 \\
\hline
\end{tabular}

Parameters for the FEM model that were invariant in the simulations made relevant to this research. 
Table S2. Ranges of parameters used in FEM simulations

\begin{tabular}{|c|c|}
\hline Feature & Range \\
\hline Periodicity $(p)$ & $10 \sim 30 \mu \mathrm{m}$ \\
\hline Length $(l)$ & $15 \sim 50 \mu \mathrm{m}$ \\
\hline Diameter $(d)$ & $0.5 \sim 8 \mu \mathrm{m}$ \\
\hline$\eta($ vs. RHE) & $-0.7 \sim-0.1 \mathrm{~V}$ \\
\hline$i_{1,0, \mathrm{NRR}}$ & $10^{-8} \sim 10^{-3} \mathrm{~mA} / \mathrm{cm}^{2}$ \\
\hline$i_{2,0, \mathrm{NRR}}$ & $10^{-8} \sim 10^{-3} \mathrm{~mA} / \mathrm{cm}^{2}$ \\
\hline$i_{1,0 \text { HER }}$ & $10^{-8} \sim 10^{-3} \mathrm{~mA} / \mathrm{cm}^{2}$ \\
\hline$i_{2,0, \mathrm{HER}}$ & $10^{-8} \sim 10^{-3} \mathrm{~mA} / \mathrm{cm}^{2}$ \\
\hline
\end{tabular}

Ranges of parameters used in the establishment of the FEM-based database. Range values are inclusive of the stated boundary values. For convenience all of our machine-learning studies arbitrarily operate with a geometric resolution of $1 \mu \mathrm{m}$, which can be improved in future investigations 
Table S3. Hyperparameters of trained MLPNNs

\begin{tabular}{|c|c|c|}
\hline Feature & Default & Chosen \\
\hline Hidden Layer Sizes & 100 & 2000 \\
\hline Activation Function & relu & relu \\
\hline Solver & adam & adam \\
\hline Validation Fraction & 0.1 & 0.3 \\
\hline Early Stopping & false & true \\
\hline
\end{tabular}

Hyperparameters of MLPNNs used for predictions. Those shown are either changed from default values or are fundamental to the machine learning model's architecture. The F.E. MLPNN was trained and optimized first (activation function, solver, hidden layer sizes, and epsilon focus for grid search). Once established, the parameters of the FE model were used as a basis for the $i_{\text {total }}$ MLPNN. Upon initial utilization, it was found that these hyperparameters were satisfactory for prediction of $i_{\text {total }}$ as shown in Figure 1F, and thus the both models had the same hyperparameters. 
Table S4. Computer hardware

\begin{tabular}{|c|c|c|}
\hline Device & Specifications & Uses \\
\hline $\begin{array}{c}\text { PC } \\
\text { (Laptop) }\end{array}$ & $\begin{array}{l}\text { Processor: } 1.4 \mathrm{GHz} \text { Dual-Core Intel Core i5 } \\
\text { Memory: } 8 \mathrm{~GB}\end{array}$ & $\begin{array}{l}\text { Predictions for electrodes using } \\
\text { trained neural networks, } \\
\text { development of figures and code } \\
\text { development. }\end{array}$ \\
\hline Workstation & $\begin{array}{l}\text { Processor: Intel® } \text { Core }^{\mathrm{TM}} \text { i7-7800X CPU @, } \\
\text { 3.50 GHz } \\
\text { Memory: } 32 \mathrm{~GB} \\
\text { GPU: NVIDIA GeForce GTX } 1060 \text { 3GB }\end{array}$ & Used for FEM simulations. \\
\hline $\begin{array}{l}\text { Virtual } \\
\text { Machine }\end{array}$ & Variable Specifications & $\begin{array}{l}\text { A virtual machine run through } \\
\text { Google Cloud Platform's } \\
\text { Jupyter Notebooks was used for } \\
\text { selecting and training machine } \\
\text { learning algorithms. }\end{array}$ \\
\hline
\end{tabular}

Details of hardware used throughout the course of this research. 
Table S5. Circled electrodes from figure $2 \mathrm{~A}$

\begin{tabular}{|c|c|c|c|c|c|c|c|c|c|c|}
\hline entry & $\begin{array}{c}p \\
(\mu \mathrm{m})\end{array}$ & $\begin{array}{c}l \\
(\mu \mathrm{m})\end{array}$ & $\begin{array}{c}d \\
(\mu \mathrm{m})\end{array}$ & $\begin{array}{c}\eta \\
(\mathrm{V})\end{array}$ & $\begin{array}{c}i_{1,0, \mathrm{NRR}} \\
\left(\mathrm{mA} / \mathrm{cm}^{2}\right)\end{array}$ & $\begin{array}{c}i_{2,0, \mathrm{NRR}} \\
\left(\mathrm{mA} / \mathrm{cm}^{2}\right)\end{array}$ & $\begin{array}{c}i_{1,0, \mathrm{HER}} \\
\left(\mathrm{mA} / \mathrm{cm}^{2}\right)\end{array}$ & $\begin{array}{c}i_{2,0, \mathrm{HER}} \\
\left(\mathrm{mA} / \mathrm{cm}^{2}\right)\end{array}$ & $\begin{array}{c}F . E . \\
(\%)\end{array}$ & $\begin{array}{c}i_{\text {total }} \\
\left(\mathrm{mA} / \mathrm{cm}^{2}\right)\end{array}$ \\
\hline 1 & 9 & 42 & 5 & -0.5 & $10^{-4}$ & $10^{-8}$ & $10^{-6}$ & $10^{-3}$ & 90.3 & -2.1 \\
\hline 2 & 10 & 48 & 8 & -0.7 & $10^{-3}$ & $10^{-3}$ & $10^{-8}$ & $10^{-8}$ & 91.0 & -2.1 \\
\hline 3 & 11 & 39 & 8 & -0.7 & $10^{-3}$ & $10^{-3}$ & $10^{-8}$ & $10^{-8}$ & 93.0 & -2.0 \\
\hline 4 & 10 & 33 & 8 & -0.7 & $10^{-3}$ & $10^{-3}$ & $10^{-8}$ & $10^{-8}$ & 94.3 & -2.0 \\
\hline
\end{tabular}

Morphological and kinetic information for circled catalysts in Figure 2A. All exchange current density and total current values have units of $\mathrm{mA} / \mathrm{cm}^{2}$ and potential is vs. RHE. The entry values correspond to the circled catalysts from left to right (lowest to highest $F$.E.). Note for convenience all of our search of optimized geometry arbitrarily operate with a geometric resolution of $1 \mu \mathrm{m}$, which can be improved in future investigations. 
Table S6. Comparison of MLPNN selected electrodes and their literature counterparts

\begin{tabular}{|c|c|c|c|c|}
\hline & \multicolumn{2}{|c|}{ Literature reported values } & \multicolumn{2}{|c|}{ MLPNN optimized } \\
\hline Catalyst & F.E. $(\%)$ & $\left|i_{\text {total }}\right|\left(\mathrm{mA} / \mathrm{cm}^{2}\right)$ & F.E. $(\%)$ & $\left|i_{\text {total }}\right|\left(\mathrm{mA} / \mathrm{cm}^{2}\right)$ \\
\hline \multirow{2}{*}{$\mathrm{Au} / \mathrm{CeO}_{\mathrm{x}}[7]$} & \multirow{2}{*}{10.10} & \multirow{2}{*}{0.13} & 17.0 & 0.16 \\
\hline & & & $\eta:-0.3 \mathrm{~V}$ & $: 80 \mu \mathrm{m}, d: 0.4 \mu \mathrm{m}$ \\
\hline \multirow{2}{*}{$\mathrm{Au} / \mathrm{TiO}_{2}[8]$} & \multirow{2}{*}{8.11} & \multirow{2}{*}{0.56} & 15.1 & 1.35 \\
\hline & & & $\eta:-0.3 \mathrm{~V}, 1$ & $l: 80 \mu \mathrm{m}, d: 10 \mu \mathrm{m}$ \\
\hline \multirow{2}{*}{$\mathrm{Au}_{6} / \mathrm{Ni}[9]$} & \multirow{2}{*}{67.8} & \multirow{2}{*}{0.21} & 99.0 & 0.37 \\
\hline & & & $\eta:-0.3 \mathrm{~V}, p$ & $l: 16 \mu \mathrm{m}, d: 8.5 \mu \mathrm{m}$ \\
\hline \multirow{2}{*}{$\begin{array}{c}\text { Au } \\
\text { Flowers [10] }\end{array}$} & \multirow{2}{*}{6.05} & \multirow{2}{*}{1.2} & 11.7 & 1.53 \\
\hline & & & $\eta:-0.3 \mathrm{~V}, p$ & $l: 80 \mu \mathrm{m}, d: 2.5 \mu \mathrm{m}$ \\
\hline \multirow{2}{*}{$\mathrm{Au} / \mathrm{NDPCs}$ [11] } & \multirow{2}{*}{12.3} & \multirow{2}{*}{0.34} & 22.7 & 0.42 \\
\hline & & & $\eta:-0.3 \mathrm{~V}$, & $l: 80 \mu \mathrm{m}, d: 10 \mu \mathrm{m}$ \\
\hline
\end{tabular}

Comparison of FE and $i_{\text {total }}$ values for reported gold catalysts and those predicted by the MLPNNs with one prediction to maximize FE and one to maximize $i_{\text {total }}$. All literature values are FE maximized and reported at $-0.2 \mathrm{~V}$ vs. RHE with the exception of $\mathrm{Au}_{6} / \mathrm{Ni}$ which was reported at $-0.14 \mathrm{~V}$ vs. RHE. Under the FE and $i_{\text {total }}$ values are summaries of relevant parameters: E, $\eta(\mathrm{V})$ vs. RHE; $p$, periodicity $(\mu \mathrm{m}) ; l$, length $(\mu \mathrm{m}) ; d$, diameter $(\mu \mathrm{m})$. 


\section{References}

1. Li, Y.; Zheng, J.; Lyu, Y.; Qiao, M.; Veder, J. P.; Marco, R. D.; Bradley, J.; Wang, R.; Huang, A.; Jiang, S. P.; Wang, S. Electron localization of gold in control of nitrogen-to-ammonia fixation. Angew. Chem. Int. Ed., 2019, 58 (51), 18604-18609.

2. Ferrell, R. T.; Himmelblau, D. M. Diffusion coefficients of nitrogen and oxygen in water. $J$. Chem. Eng. Data 1967, 12 (1), 111-115.

3. Chen, H.; Voth, G. A.; Agmon, N. Kinetics of Proton Migration in Liquid Water. J. Phys. Chem. B 2010, 114 (1), 333-339.

4. Kolev, N. Multiphase Flow Dynamics 4: Turbulence, Gas Adsorption and Release, Diesel Fuel Properties. Springer, Berlin, Heidelberg, Germany: 2012.

5. Nørskov, J. K.; Bligaard, T.; Logadottir, A.; Kitchin, J. R.; Chen, J. G.; Pandelov, S.; Stimming, U. Trends in the Exchange Current for Hydrogen Evolution. J. Electrochem. Soc. 2005, 152 (3), J23-J26.

6. Seh, Z. W.; Kibsgaard, J.; Dickens, C. F.; Chorkendorff, I.; Nørskov, J. K.; Jaramillo, T. F. Combining theory and experiment in electrocatalysis: Insights into materials design. Science 2017, 355 (6321), eaad4998.

7. Li, S.-J.; Bao, D.; Shi, M.-M.; Wulan, B.-R.; Yan, J.-M.; Jiang, Q. Amorphizing of Au Nanoparticles by $\mathrm{CeO}_{x}-\mathrm{RGO}$ Hybrid Support towards Highly Efficient Electrocatalyst for $\mathrm{N}_{2}$ Reduction under Ambient Conditions. Adv. Mater. 2017, 29 (33), 1700001.

8. Shi, M.-M.; Bao, D.; Wulan, B.-R.; Li, Y.-H.; Zhang, Y.-F.; Yan, J.-M.; Jiang, Q. Au SubNanoclusters on $\mathrm{TiO}_{2}$ toward Highly Efficient and Selective Electrocatalyst for $\mathrm{N}_{2}$ Conversion to $\mathrm{NH}_{3}$ at Ambient Conditions. Adv. Mater. 2017, 29 (17), 1606550.

9. Xue, Z.-H.; Zhang, S.-N.; Lin, Y.-X.; Su, H.; Zhai, G.-Y.; Han, J.-T.; Yu, Q.-Y.; Li, X.-H.; Antonietti, M.; Chen, J.-S. Electrochemical Reduction of $\mathrm{N}_{2}$ into $\mathrm{NH}_{3}$ by Donor-Acceptor Couples of $\mathrm{Ni}$ and $\mathrm{Au}$ Nanoparticles with a 67.8\% Faradaic Efficiency. J. Am. Chem. Soc. 2019, 141 (38), 14976-14980.

10. Wang, Z.; Li, Y.; Yu, H.; Xu, Y.; Xue, H.; Li, X.; Wang, H.; Wang, L. Ambient Electrochemical Synthesis of Ammonia from Nitrogen and Water Catalyzed by Flower-Like Gold Microstructures. ChemSusChem 2018, 11 (19), 3480-3485.

11. Qin, Q.; Heil, T.; Antonietti, M.; Oschatz, M. Single-Site Gold Catalysts on Hierarchical NDoped Porous Noble Carbon for Enhanced Electrochemical Reduction of Nitrogen. Small Methods 2018, 2 (12), 1800202. 